\title{
A Practical Solution for Torsional Vibrations Evasion in Variable Speed Drives
}

\author{
Khaled ElShawarby, Roberto Perini, \\ Giovanni M. Foglia, Antonino Di Gerlando \\ $D E N G$ \\ Politecnico di Milano \\ Milan, Italy \\ $\{$ khaled.elshawarby, roberto.perini \\ gianmaria.foglia, antonino.digerlando\}@polimi.it
}

\author{
Mattia Rossi, Francesco Castelli Dezza \\ $D M E C$ \\ Politecnico di Milano \\ Milan, Italy \\ \{mattia.rossi, francesco.castellidezza\}@ polimi.it
}

\begin{abstract}
The paper presents a practical solution for an existing drive-train to avoid torsional vibrations for variable speed drives under SPWM modulation technique. Torsional natural frequencies can be a problem because either ill defined in design stage or one of the mechanical parameters has changed after the initial design stage. The proposed method comprises of dividing the frequency range into several partitions where in each partition a value of modulation frequency ratio $m_{f}$ is chosen. A criterion is developed to choose $m_{f}$ based on avoiding torsional excitations with the minimum converter losses. Torque harmonic distortion and converter losses are analyzed. Matlab/Simulink is used to validate the approach.
\end{abstract}

Index Terms-Torsional Vibrations, Variable Speed Drives, Sinusoidal Pulse Width Modulation (SPWM).

\section{INTRODUCTION}

One of the technical challenges associated with electric machines driven by power electronics converters is the mitigation of torsional vibrations. Torsional resonances occur for any drive-train; even simple drive trains consisting of a motor driving a load have at least one torsional mode. Converter modulation techniques generate torque harmonics which subsequently excite the natural resonance frequency of the drive-train. Torque harmonics amplitudes as low as $1 \%$ of the rated torque can be dangerous [1]. Torsional vibrations is even a bigger concern for high power applications thus various mitigation techniques have been studied [2]-[7].

This paper proposes a practical solution to avoid torsional vibrations in variable speed existing applications, also maintaining low switching losses and better quality waveforms. The principle of changing modulation frequency ratio $m_{f}$ for each frequency range is a well known concept in the literature. The proposal is to use it to eliminate torsional vibration problems.

Wind Energy Conversion Systems (WECSs) can be suited for this kind of approach because wind power harvesting is achieved in a defined wind speed range namely from cutin speed $V_{w, c u t-i n}$ up to the rated speed $V_{w, \text { rated }}$ as shown in Fig. 1. Thus, a starting frequency ratio $m_{f o}$ can be of a value high enough in a way that no critical points can be excited. Since the dynamical phenomenon involved in WECSs application is slow, the operating frequency range can be

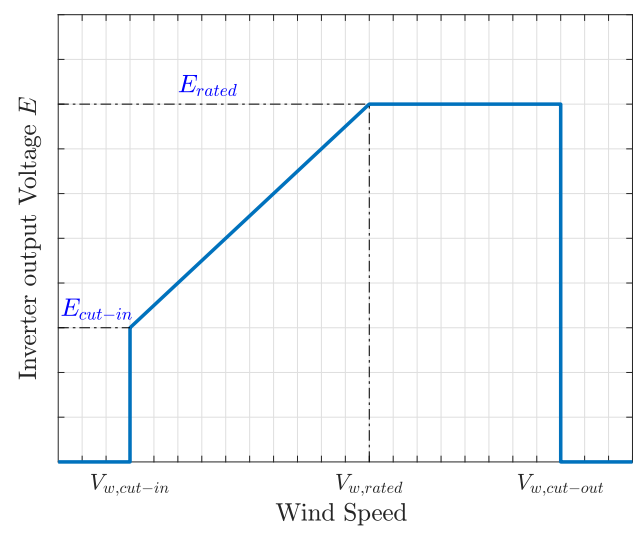

Fig. 1. Typical wind energy converter output voltage. The converter harnesses power from $V_{w, c u t-i n}$ to $V_{w, c u t-o u t}$, where the linear range $\left(V_{w, c u t-i n}\right.$ to $\left.V_{w, \text { rated }}\right)$ is run usually under MPPT conditions.

divided into portions: in each portion a selected $m_{f}$ is chosen. The criteria of selecting $m_{f}$ can be simplified into two points:

- avoiding critical frequencies excitation;

- keeping switching frequency fixed in a certain range, i.e. limited switching losses.

The rest of this work is organized as follows. Section II defines the method to divide the frequency regions and the choice of $m_{f}$ with respect to torsional vibrations excitation. Section III introduces the system to be studied, focuses on the mechanical assembly, defining the torsional natural frequencies. Section IV derives the approximated expression of the electromagnetic torque for SPWM modulation. Section V analyzes the converter losses and the torque total harmonic distortion for the proposed method. Section VI presents simulations results of the proposed technique.

\section{FREQUENCY RANGE DEFINITION AND CRITERIA TO CHOOSE $m_{f}$}

\section{A. Criteria to choose $m_{f}$}

The choice of $m_{f}$ is based on avoiding torsional vibrations excitation keeping $m_{f}$ odd and multiple of three. In 
order to avoid critical points, a Campbell diagram should be considered. It describes the relation between electrical harmonic excitations versus the torsional natural frequencies. The intersections between a harmonic frequency and one of the torsional natural frequencies $T N F_{i}$ represent the so called critical point i.e. torsional resonance occurrence. A critical frequency $f_{c r}$ can be found by dividing $T N F_{i}$ by a random harmonic $h$.

Considering Sinusoidal PWM (SPWM) technique, according to the analysis performed in [8] for the electromagnetic torques, the least order harmonic present $h=m_{f}-3$. In order to avoid the harmonic excitation, $m_{f}$ can be calculated as:

$$
m_{f} \geq \frac{T N F_{\max }}{f_{\text {limit }}}+3
$$

where $f_{\text {limit }}$ is the lowest starting frequency to avoid critical points (critical points occur for frequenices lower than $f_{\text {limit }}$ ). In order to keep low switching losses, (1) has to be minimized keeping $m_{f}$ odd and multiple of three.

\section{B. Frequency Range Definition}

Thus, in order to avoid the torsional vibrations excitations, it is enough to set $f_{\text {limit }}$ equal to $f_{c u t-i n}$ and evaluate $m_{f}$ accordingly. However, it is not necessary to operate at high switching frequencies (i.e. higher converter losses) for a wide frequency range. Thus, a way can be dividing the speed range into partitions in which each partition lower frequency will be a new $f_{\text {limit }}$ entailing a new value of $m_{f}$.

There can be several aspects to consider in how to divide the operating range of a certain application since it depends on the application itself. As for WECSs application, the idea is to divide the frequency range, based on the frequency of occurrence, as shown in Fig. 2 into three portions:

- near cut-in frequency region $\left(f_{\text {cut-in }}-f_{\text {limit }_{1}}\right)$;

- an intermediate frequency region $\left(f_{\text {limit }_{1}}-f_{\text {limit }_{2}}\right)$;

- near rated frequency region $\left(f_{\text {limit } 2}-f_{\text {rated }}\right)$.

It has to be noted that, if the working frequency is fluctuating around a border frequency limit $f_{\text {limit }_{n}}, m_{f}$ will fluctuate as well between two values. Thus, some constraints have to be set in order to avoid such condition:

- changing $m_{f}$ according to a tolerance band;

- changing $m_{f}$ if the frequency stabilizes for a given time.

Some additional constraints can be considered to the evasion of torsional vibration as a defined maximum level of converter losses or a maximum value of the total harmonic distortion $T H D$. In such a case, the frequency range should be divided into smaller portions where for each of them a new $m_{f}$ is chosen. However, the number of transients will increase due to the frequent change of $m_{f}$.

\section{Mechanical Assembly}

Consider a three-modules Axial Flux Permanent Magnet Synchronous Generator (AFPMSG) drive train as shown in Fig. 3 whose data are reported in Table I. The AFPMSG is directly driven (DD) by a wind turbine whose inertia is

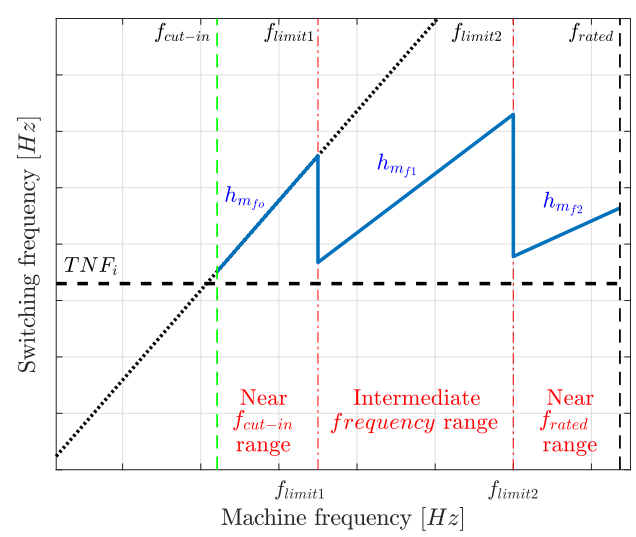

Fig. 2. Campbell diagram for the proposed method of dividing the frequency range into portions: Near $f_{c u t-i n}$ - Intermediate frequency - Near $f_{\text {rated }}$ ranges, against operating with $m_{f}$ high enough to avoid $T N F_{i}$. In each region $m_{f}$ decreases, i.e. the corresponding harmonic $h$ has a lower slope.

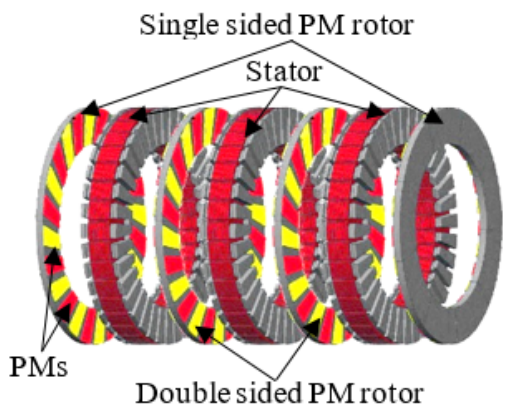

(a)

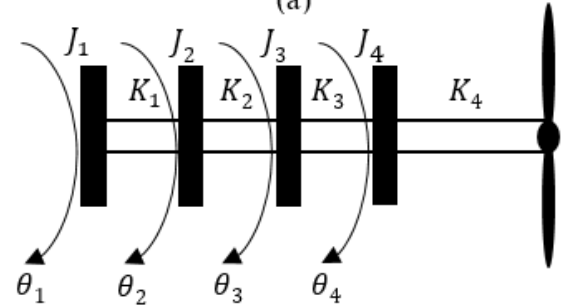

(b)

Fig. 3. (a) Three-module AFPMSG consists of three stators and four rotors where the outer rotor disks $\left(J_{1}, J_{4}\right)$ are single-sided PM rotors, while both middle rotor disks $\left(J_{2}, J_{3}\right)$ are double sided PM rotors (b) Mechanical equivalent model for the machine connected to the wind turbine.

much higher than any of the machine rotors. Thus, the angular deflection of the turbine is assumed to be zero.

Using the well known eigen-values approach, natural frequencies can be calculated by equating the Newton's equations to zero. Assuming Simple harmonic motion and neglecting the internal damping, the mechanical system of equations can be written as:

$$
\left((2 \pi *\{T N F\})^{2}[J]+[K]\right) *[\theta]=\{0\}
$$

where $T N F$ is a vector of the torsional natural frequencies of the mechanical assembly. $[J]$ is a diagonal matrix of the moments of inertia of the rotors: $J_{1}$ and $J_{4}$ are both single- 

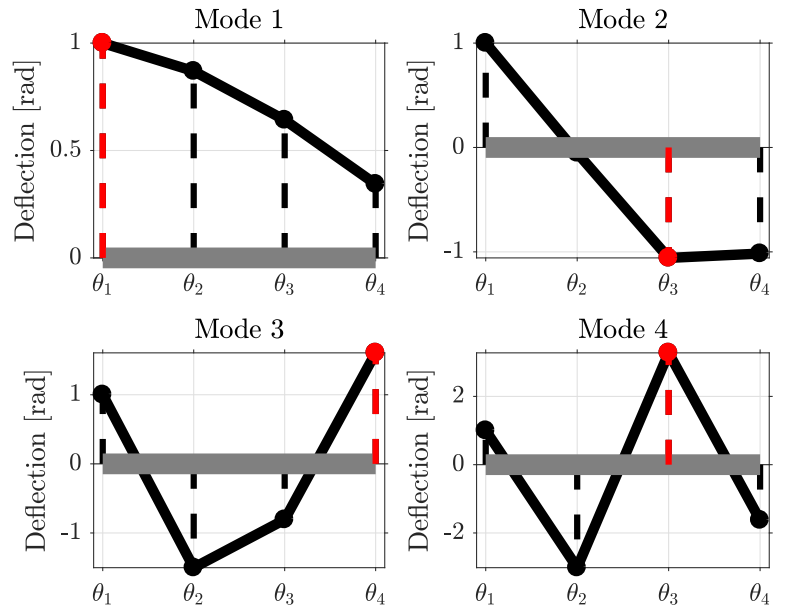

Fig. 4. Angular deflection of each rotor with respect to each mode of vibration. The angular deflection $\theta_{1}$ is taken as a reference $\theta_{1}=1$ [rad]. The maximum angular deflection for each mode is highlighted in red which will be used in the following analysis.

sided permanent magnets (PMs) rotors while $J_{2}$ and $J_{3}$ are double-sided PMs rotors. $[K]$ is the stiffness matrix and is given by:

$$
[K]=\left[\begin{array}{cccc}
K_{1} & -K_{1} & 0 & 0 \\
-K_{1} & K_{1}+K_{2} & -K_{2} & 0 \\
0 & -K_{2} & K_{2}+K_{3} & -K_{3} \\
0 & 0 & -K_{3} & K_{3}+K_{4}
\end{array}\right]
$$

where each individual stiffness is calculated using the classical De Saint Venant's principles. The values are verified to be an accurate approximation compared to the values obtained from Finite Element Method (FEM) with ANSYS Mechanical. $[\theta]$ is the vector of angular deflection of the four rotors. The shaft portion between the machine and turbine $K_{4}$ is assumed to have the same stiffness as the one of the internal machine shaft portions.

Thus, solving (2), the natural frequencies of the system are:

$$
T N F=\left\{\begin{array}{c}
239 \\
679 \\
1047 \\
1326
\end{array}\right\}[\mathrm{Hz}]
$$

Each Natural frequency corresponds to a mode of vibration as shown in Fig. 4. However, the lower modes of vibrations are always more critical with respect to higher modes due to their low inherent damping. Thus, for the purpose of the analysis, only the first and second modes are taken into account.

\section{Electric Excitations due to SPWM}

An approximate mathematical expression was provided in [7], which accurately calculates the electromagnetic torque $\left(T_{e}\right)$ harmonic amplitudes due to the SPWM modulation. The torsional vibrations in AFPMSG is a local phenomenon occurring on the shaft connecting two rotors. Thus, the considered
TABLE I

SINGLE MODULE AXIAL FLUX PERMANENT MAGNET MACHINE NAMEPLATE DATA

\begin{tabular}{|l|c|}
\hline$P_{n}[\mathrm{MW}], I_{n}[\mathrm{~A}]$ & $1.0,713$ \\
\hline Rated frequency $f_{\text {rated }}[\mathrm{Hz}]$, Rated Speed $\Omega_{n}[\mathrm{rpm}]$ & $14.73,17$ \\
\hline $\begin{array}{l}\text { Line to neutral EMF at rated speed }[\mathrm{V}] \\
\text { (sinusoidal waveform) }\end{array}$ & 435 \\
\hline Phase resistance $R[\mathrm{~m} \Omega]$ & 14.59 \\
\hline Synchronous inductance $(d, q$ axes) $L[\mathrm{mH}]$ & 4.321 \\
\hline Rotor ext. diameter $R_{\text {ext }}$, Axial length $[\mathrm{m}]$ & $5.00,0.603$ \\
\hline Hollow shaft stiffness $K[\mathrm{Nm} / \mathrm{rad}]$ & $8.93 * 10^{11}$ \\
\hline Single-sided PM rotors inertias $J_{1}, J_{4}\left[\mathrm{kgm}^{2}\right]$ & $5.17 * 10^{4}$ \\
\hline Double-sided PM rotors inertias $J_{2}, J_{3}\left[\mathrm{kgm}^{2}\right]$ & $4.4 * 10^{4}$ \\
\hline Turbine inertia $\left[\mathrm{kgm}^{2}\right]$ & $3 * 10^{6}$ \\
\hline No damping cage & \\
\hline
\end{tabular}

$T_{e}$ is the one $T_{e_{\mu}}$ produced by each module $\mu$ of the machine

$$
T_{e_{\mu}}=\frac{\sqrt{3} * p * \psi_{P M}}{2} i_{q_{\mu}}
$$

where, $p$ is the number of poles, $\psi_{P M}$ is the PM flux and $i_{q_{\mu}}$ is the $\mu$-th module quadrature current, which is given by (5).

$$
\begin{aligned}
i_{q \mu}(t) & =\sqrt{\frac{3}{2}} \cdot\left[I_{1} \sin \left(\phi+\delta-\frac{\pi}{2}\right)\right. \\
& +I_{m_{f}-4} \sin \left(\left(m_{f}-3\right) \omega_{\mathrm{o}} \mathrm{t}+\delta-\theta_{m_{f}-4}-\frac{\pi}{2}\right) \\
& -I_{m_{f}-2} \sin \left(\left(m_{f}-3\right) \omega_{\mathrm{o}} \mathrm{t}-\delta-\theta_{m_{f}-2}+\frac{\pi}{2}\right) \\
& +I_{m_{f}+2} \sin \left(\left(m_{f}+3\right) \omega_{\mathrm{o}} \mathrm{t}+\delta-\theta_{m_{f}+2}-\frac{\pi}{2}\right) \\
& -I_{m_{f}+4} \sin \left(\left(m_{f}+3\right) \omega_{\mathrm{o}} \mathrm{t}-\delta-\theta_{m_{f}+4}+\frac{\pi}{2}\right) \\
& +I_{2 m_{f}-1} \sin \left(\left(2 m_{f}\right) \omega_{\mathrm{o}} \mathrm{t}+\delta-\theta_{2 m_{f}-1}-\frac{\pi}{2}\right) \\
& -I_{2 m_{f}+1} \sin \left(\left(2 m_{f}\right) \omega_{\mathrm{o}} \mathrm{t}-\delta-\theta_{2 m_{f}+1}+\frac{\pi}{2}\right) \\
& +I_{3 m_{f}-4} \sin \left(\left(3 m_{f}-3\right) \omega_{\mathrm{o}} \mathrm{t}+\delta-\theta_{3 m_{f}-4}-\frac{\pi}{2}\right) \\
& -I_{3 m_{f}-2} \sin \left(\left(3 m_{f}-3\right) \omega_{\mathrm{o}} \mathrm{t}-\delta-\theta_{3 m_{f}-2}+\frac{\pi}{2}\right) \\
& +I_{3 m_{f}+2} \sin \left(\left(3 m_{f}+3\right) \omega_{\mathrm{o}} \mathrm{t}+\delta-\theta_{3 m_{f}+2}-\frac{\pi}{2}\right) \\
& -I_{3 m_{f}+4} \sin \left(\left(3 m_{f}+3\right) \omega_{\mathrm{o}} \mathrm{t}-\delta-\theta_{3 m_{f}+4}+\frac{\pi}{2}\right) \\
& +I_{4 m_{f}-1} \sin \left(\left(4 m_{f}\right) \omega_{\mathrm{o}} \mathrm{t}+\delta-\theta_{4 m_{f}-1}-\frac{\pi}{2}\right) \\
& \left.-I_{4 m_{f}+1} \sin \left(\left(4 m_{f}\right) \omega_{\mathrm{o}} \mathrm{t}-\delta-\theta_{4 m_{f}+1}+\frac{\pi}{2}\right)\right]
\end{aligned}
$$

where, $I_{h}$ is the current harmonic amplitude, $\phi$ the displacement between voltage and current fundamental components, $\delta$ the load angle, $m_{f}$ the SPWM frequency modulation ratio, $\omega_{o}$ the output fundamental angular frequency. The voltage and current harmonic amplitudes and characteristic angles, $I_{h}$ and $\theta_{h}$, are obtained by applying each voltage harmonic, calculated in [8], to the equivalent $R-L-e$ circuit of the AFPMSG. 
TABLE II

VALUES OF $m_{f}$ WHICH AVOIDS THE NATURAL RESONANCES FOR BOTH $T N F_{1}$ AND $T N F_{2}$

\begin{tabular}{|c|c|c|c|}
\hline & $\begin{array}{c}f_{\text {cut }_{\text {in }}} \text { to } \\
f_{\text {limit }_{1}}\end{array}$ & $\begin{array}{c}f_{\text {limit }_{1}}- \\
f_{\text {limit }_{2}}\end{array}$ & $\begin{array}{c}f_{\text {limit }_{2}}- \\
f_{\text {rated }}\end{array}$ \\
\hline$m_{f, T N F 1}$ & 63 & 39 & 27 \\
\hline$m_{f, T N F 2}$ & 159 & 105 & 63 \\
\hline
\end{tabular}

From (4) and (5), the torque $T H D$ for the $\mu$-th module $T H D_{T \mu}$ can be evaluated; it is evident the dependence $T H D_{T \mu}$ on the ac current harmonic amplitudes $I_{h}$.

\section{HARMONiC Distortion ANd CONVERTER Losses ANALYSIS}

The proposed method is mainly focused on avoiding $T N F$ excitations and on dividing the frequency range into portions in order to decrease $m_{f}$. Basically that has consequences on the converter losses and $T H D_{T}$. Assuming $f_{\text {cut-in }}=0.3 \cdot f_{\text {rated }}$ $=4.42 \mathrm{~Hz}$, the limit frequencies are assumed to be 7.5 and $11.5 \mathrm{~Hz}$, then two cases are considered: the former avoids the excitation of the $T N F_{1}$, while the latter avoids the excitation of both $T N F_{1}$ and $T N F_{2}$. For each case the values of $m_{f}$ are calculated by choosing the first odd $m_{f}$ and multiple of three satisfying (1) and are labeled as $m_{f, T N F 1}$ and $m_{f, T N F 2}$.

Fig. 5 shows the Campbell diagram for $m_{f, T N F 1}$ and $m_{f, T N F 2}$. In particular the least order harmonic $m_{f}-3$ is plotted for each frequency range. It can be seen no critical points are excited for the targeted $T N F$.It has to be noted that only $m_{f}-3$ are plotted which means higher order harmonics will probably excite higher modes. As an example, Fig. 6 shows the effect of high order harmonics for $m_{f, T N F 1}$ on $T N F 2$. It shows the harmonic $2 m_{f}$ exciting $T N F_{2}$ in each frequency partition, i.e. three critical frequencies arise $\left(f_{c r 1}\right.$, $\left.f_{c r 2}, f_{c r 3}\right)$. The amplitudes of the angular deflection due to vibrations depend on the amplitude of the exciting torque and the internal damping of the system. Thus, it has to be verified that the excitation of these critical points does not cause problems for the system.

Fig. 7 shows the effect of such a combination of $m_{f}$ values on the $T H D_{T}$. Two issues are apparent [8], [7]: if $m_{f}$ increases, the harmonic order $h$ increases, and the harmonic amplitude $I_{h}$ decreases according to (6)

$$
I_{h}=\frac{V_{h}}{Z_{h}}=\frac{V_{h}}{\sqrt{R^{2}+\left(h \cdot \omega_{o} \cdot L\right)^{2}}}
$$

where, $V_{h}$ and $Z_{h}$ are the harmonic voltages and characteristic impedances respectively. As mentioned earlier, $T H D_{T} \propto$ $I_{h}$, Therefore, $T H D_{T} \propto m_{f}{ }^{-1}$. Moreover, at low frequencies, the $T H D_{T}$ increases as the weight of the harmonics to the fundamental increases.

The used converter comprises of six IGBTs where the total converter losses $\left(P_{\text {total }}\right)$ can be split into conduction and commutation losses. Conduction losses occur in the diodes and transistors and are denoted by $P_{D}$ and $P_{T}$ respectively. While transistor commutation losses are function of the switching frequency and denoted by $P_{C O M}$. For SPWM modulation the

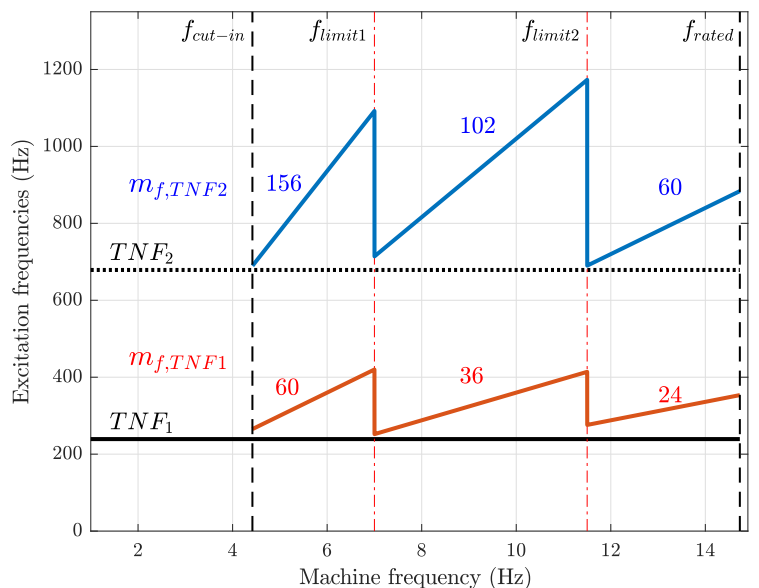

Fig. 5. Campbell Diagram for the proposed method to avoid $T N F_{1}$ (solid) and $T N F_{2}$ (dotted). The only harmonic shown is the least $m_{f}-3$. It can be seen there are no critical points excited, i.e. no resonance occurs.

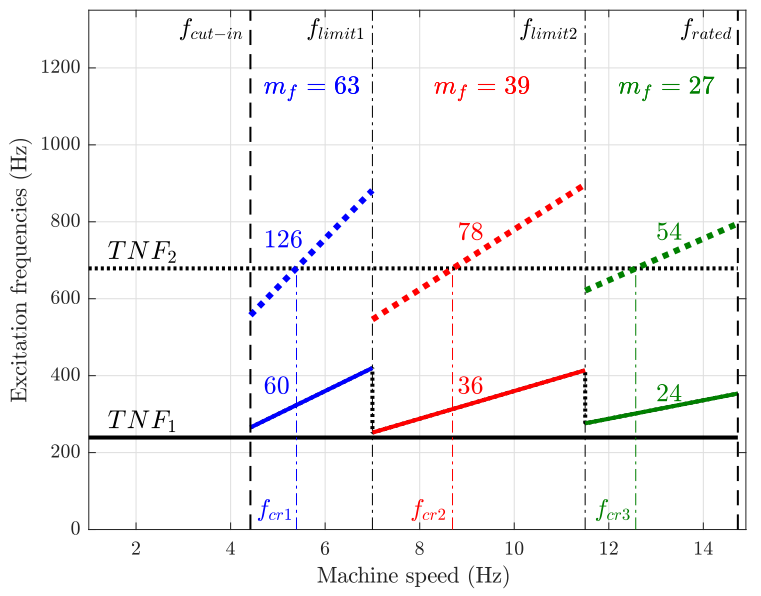

Fig. 6. The Campbell diagram of the proposed method adopting the first $T N F$ as a reference to avoid. As it can be seen some critical points arises from the intersection of the harmonic $2 m_{f}$ with the second $T N F$

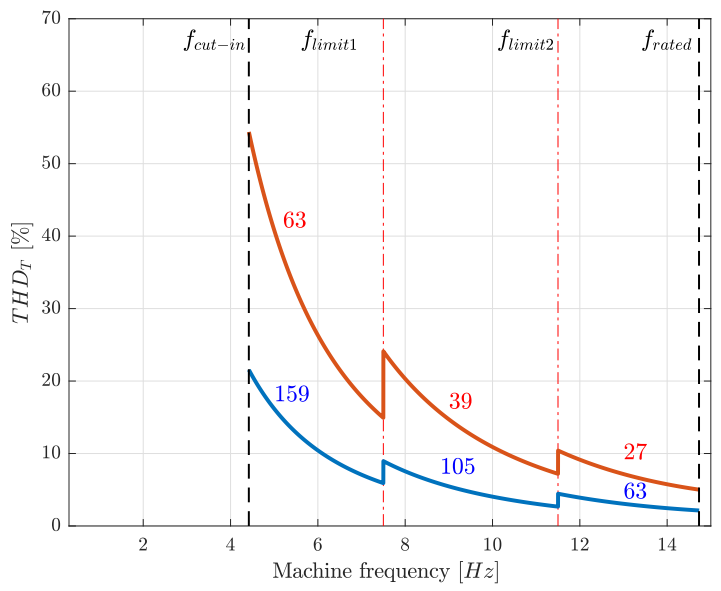

Fig. 7. $T H D_{T}$ for the proposed method to avoid $T N F_{1}$ and $T N F_{2}$. Using higher values of $m_{f}$ has lower values of the harmonic distortion. 


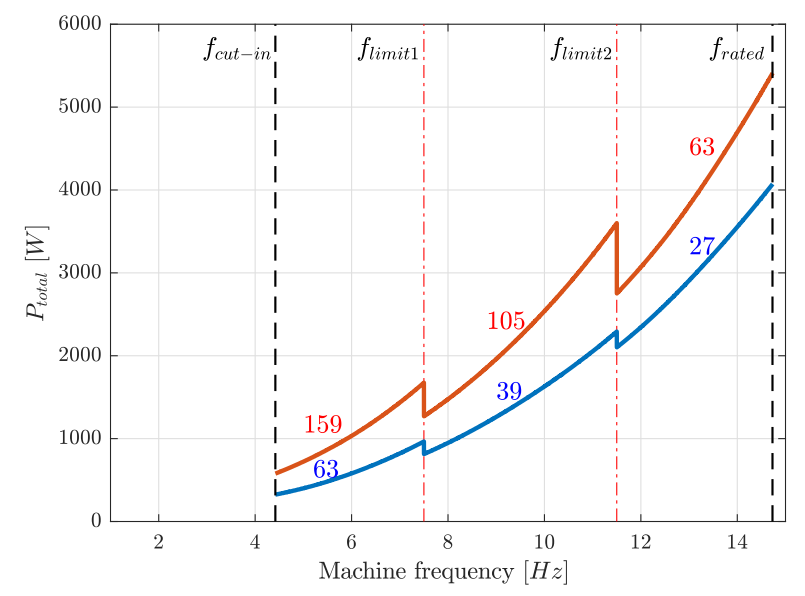

Fig. 8. Single module converter losses $P_{\text {total }}$ for the proposed method to avoid both $T N F_{1}$ and $T N F_{2}$. High $m_{f}$ implies high switching losses.

average conduction losses over the fundamental period $T$ can be calculated as:

$$
\begin{array}{r}
P_{D}=\frac{1}{T} \int_{0}^{\frac{T}{2}}(1-\delta(t)) \cdot\left[V_{F} \cdot i(t)+R_{D T} \cdot i^{2}(t)\right] d t \\
P_{T}=\frac{1}{T} \int_{0}^{\frac{T}{2}} \delta(t) \cdot\left[V_{C E} \cdot i(t)+R_{T T} \cdot i^{2}(t)\right] d t
\end{array}
$$

where $V_{F}$ and $V_{C E}$ are the on-state threshold voltages across the diode and collector-emitter power switch voltage respectively, $R_{D T}$ and $R_{T T}$ is the slope resistance for the diode and power switch respectively. Such parameters are obtained directly from the switch datasheet. $\delta(t)$ is the SPWM duty cycle which is given by $\left(\delta(t)=\frac{1}{2}+\frac{1}{2} M \sin \left(\omega_{o} t\right)\right)$, where $M$ is the amplitude modulation index.

$P_{C O M}$ is given by:

$$
P_{C O M}=\frac{f_{s w} \cdot V_{D C} \cdot \max (i(t))}{V_{c c} \cdot I_{c c} \cdot \pi}\left(E_{O N}+E_{O F F}\right)
$$

where, $f_{s w}$ is the switching frequency, $V_{D C}$ is the DC bus voltage, $E_{O N}$ and $E_{O F F}$ are the turn-on, turn-off switching energy losses respectively and $\max (i(t))$ is the maximum value of the ac current, $V_{c c}$ and $I_{c c}$ are the test voltage and current respectively.

Fig. 8 shows an estimation of the losses in switches $P_{\text {total }}$ for the high power switch whose data are in Table III. $P_{\text {total }}$ is calculated by summing eqs. (7) and (8) for both $m_{f, T N F 1}$ and $m_{f, T N F 2}$. Due to the dependence on $f_{s w}$, it can be noticed that $P_{\text {total }}$ is directly proportional to $m_{f}$.

\section{Simulation Results}

The system presented in Section III is implemented in Matlab/Simulink. Each module is modeled by standard two axis d-q model of PMSM. For each module the classical FOC

\begin{tabular}{|c|c|}
\hline Collector Emitter Voltage $V_{C E S}[\mathrm{~V}]$ & 1700 \\
\hline Collector Current $I_{C n}[\mathrm{~A}] @ T_{c}=100{ }^{\circ} \mathrm{C}$ & 1400 \\
\hline Turn-on, turn-off switching loss $E_{O N}, E_{O F F}[\mathrm{~mJ}]$ & 502,618 \\
\hline Diode forward voltage $V_{F}$ [V] @ $I_{F}=1400 \mathrm{~A}$ & 1.8 \\
\hline Maximum Power Dissipation @ $T_{j}=175{ }^{\circ} \mathrm{C} V_{F}[\mathrm{~kW}]$ & 9.37 \\
\hline \multicolumn{2}{|l|}{ Junction to Case Thermal Resistance } \\
\hline for IGBT $R_{t h J C_{I}}$ and diodes $R_{t h J C_{D}}[\mathrm{~K} / \mathrm{kW}]$ & 16,33 \\
\hline \multicolumn{2}{|l|}{ Case to Heat-sink Thermal Resistance } \\
\hline for IGBT $R_{t h C H_{I}}$ and diodes $R_{t h}$ & $8.9,18.4$ \\
\hline
\end{tabular}

TABLE III

High Power SwITCH $G D 1400 H F T 170 P 2 S$ RATED DATA

is implemented, the machine is vector-controlled to operate in maximum torque-per-ampere (MTPA) conditions.

For the purpose of the analysis, the system is accelerated from $f_{c u t-i n}$ to $f_{\text {rated }}$, despite the slow dynamics of the wind turbine. The angular deflection of all four rotors of the machine is observed. The first considered case is using the proposed method to avoid the excitation of $T N F_{1}$, which entitles the excitation of higher modes of vibration.

Fig. 9 shows the effect, due to the excitation of $T N F_{2}$, on the angular deflection of the third rotor $\theta_{3}$ due to the operation with $m_{f, T N F 1}$. The maximum deflection due to second mode excitation is shown in Fig. 4 is $\theta_{3}$. An amplification of $\theta_{3}$ occurs at the critical frequencies, however it remains small compared to the maximum allowable deflection which can be calculated as:

$$
\theta_{\max }=\frac{0.8 \cdot \sigma_{\max } \cdot l_{s h}}{G \cdot R_{e x t}} \simeq 4 \cdot 10^{-4} \quad[\mathrm{rad}]
$$

where, $\sigma_{\max }$ is the maximum allowable stress for steel $\simeq$ 117.7 $\mathrm{MPa}, G$ is the torsional modulus of rigidity for steel $\simeq 79.3 \mathrm{GPa}, l_{s h}$ and $R_{e x t}$ are the length of the shaft between two rotor and the rotor external radius respectively.

Fig. 10 shows the angular deflection of the maximum deflected rotor $\theta_{4}$ and $\theta_{3}$ due to the third and fourth mode excitations respectively shown in Fig. 4. Due to the operation with $m_{f, T N F 2}$, even if the higher modes are excited, the internal damping of the system is enough to maintain the deflection undetected.

Fig. 11 compares the electromagnetic torque for the operation with $m_{f, T N F 1}$ and $m_{f, T N F 2}$. As it can be seen, the ripple content for $m_{f, T N F 2}$ is much lower.

Since the proposed method mainly affects $T H D_{T}$ and $P_{\text {total }}$, the choice remains related to the application:

- If the internal damping is not high enough, higher $T N F s$ have to be considered. Thus, it will have the priority in choosing the values of $m_{f}$;

- $T H D_{T}$, which is directly proportional to $I_{h}$, can also be the defining criterion. As discussed in [9], the higher the current harmonics, the higher the dc bus voltage distortions are. As a result the expected life-time of the capacitor dielectric decreases. In addition, the higher harmonic distortions also affect the quality of the operation, may raise the temperature of the windings increasing the wear-rate of the insulation. This can be achieved by 

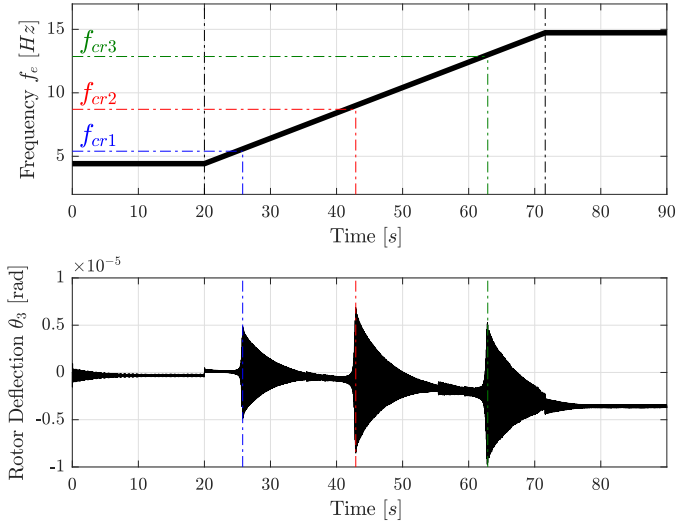

Fig. 9. Operation with $m_{f, T N F 1}$ (a) machine frequency $f_{e}$ during the acceleration from $f_{c u t-i n}$ to $f_{\text {rated }}$; (b) angular deflection of the third rotor $\theta_{3}$ being excited by $T N F_{2}$. An amplification of the deflection occurs but still remains well away from the maximum deflection $\theta_{\max }$.
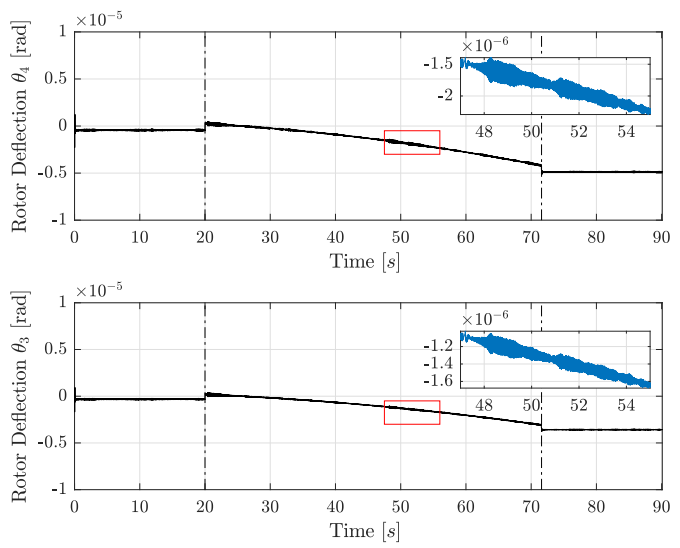

Fig. 10. Operation with $m_{f, T N F 2}$ (a) angular deflection of the fourth rotor $\theta_{4}$ excited by $T N F_{3}$; (b) angular deflection of the third rotor $\theta_{4}$ excited by $T N F_{4}$. The deflection is undetected due to the system internal damping.

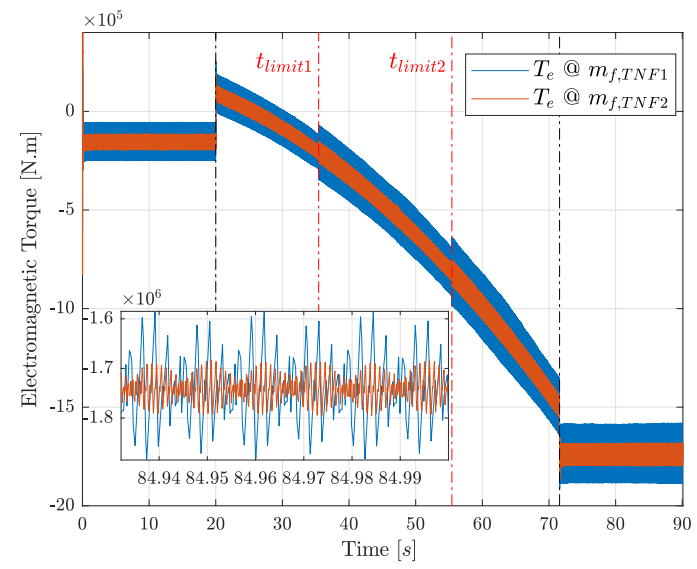

Fig. 11. Electromagnetic torque for the operation under $m_{f, T N F 1}$ and $m_{f, T N F 2}$ showing the change in $m_{f}$ at $t_{\text {limit } 1}$ and $t_{\text {limit } 2}$ (corresponding to $f_{\text {limit } 1}$ and $\left.f_{\text {limit } 2}\right)$. Also it shows the waveforms for a period at $f_{\text {rated }}$. the division of the lower frequency portions into several smaller portions changing $m_{f}$ more frequently in order to keep a maximum limit of $T H D$;

- converter losses $P_{\text {total }}$ can be the critical parameter. If the cooling system needs to be upgraded in order to dissipate more power, this implies a defined maximum limit $P_{t_{\text {total }}}$. Thus, the choice of $m_{f}$ can be calculated from setting the limit $P_{\text {total }_{m}}$ which can be done by dividing the higher frequency portions into smaller portions.

\section{CONCLUSION}

This paper proposed a practical approach to avoid torsional vibration excitations. This method can be applied for existing applications driven by $S P W M$ modulation. It consists of using a value of $m_{f}$ high enough to avoid the TNF by the least order torque harmonic $m_{f}-3$. In order to limit switching losses, the operating region is divided into several partitions. A criterion to define $m_{f}$ for each partition that also respects the aforementioned constraint was defined.

The approach was applied on AFPMSG machine consisting of four rotors directly driven by a wind turbine. Such a mechanical assembly has four modes of vibrations. Avoiding torsional excitations from the first mode or from the first and second modes were the only cases considered. An analytical analysis considering torque harmonic distortion $T H D_{T}$ and converter losses was performed for the overall speed range. Matlab/Simulink simulation validated the approach

\section{REFERENCES}

[1] J. Song-Manguelle, C. Sihler, and J. M. Nyobe-Yome, "Modeling of torsional resonances for multi-megawatt drives design," in 2008 IEEE Industry Applications Society Annual Meeting, Oct 2008, pp. 1-8.

[2] J. Song-Manguelle, J. M. N.-Yome, and G. Ekemb, "Pulsating torques in pwm multi-megawatt drives for torsional analysis of large shafts," IEEE Transactions on Industry Applications, vol. 46, no. 1, pp. 130-138, Jan 2010.

[3] M. Bruha and Z. Peroutka, "Torsional vibration in large variable speed drive systems: Origin and mitigation methods," in 2015 17th European Conference on Power Electronics and Applications (EPE'15 ECCEEurope), Sept 2015, pp. 1-10.

[4] F. Fateh, W. N. White, and D. Gruenbacher, "Torsional vibrations mitigation in the drivetrain of dfig-based grid-connected wind turbine," IEEE Transactions on Industry Applications, vol. 53, no. 6, pp. 5760-5767, Nov 2017.

[5] J. Song-Manguelle, C. Sihler, and S. Schramm, "A general approach of damping torsional resonance modes in multi-megawatt applications," in 2010 IEEE Energy Conversion Congress and Exposition, Sept 2010, pp. 772-779.

[6] Z. Q. Zhu and J. H. Leong, "Analysis and mitigation of torsional vibration of pm brushless ac/dc drives with direct torque controller," IEEE Transactions on Industry Applications, pp. 1296-1306, July 2012.

[7] A. Di Gerlando, K. ElShawarby, G. M. Foglia, and R. Perini, "Torsional vibration mitigation by harmonic inversion through spwm carrier signal control," in 2018 XIII International Conference on Electrical Machines (ICEM), Sep. 2018, pp. 2330-2336.

[8] A. Di Gerlando, K. EIShawarby, G. M. Foglia, M. F. Iacchetti, and R. Perini, "Dc current and torque ripple mitigation in modular pmsg drives for multi-mw wecss with linear pwm inverter modulation," in 2018 XIII International Conference on Electrical Machines (ICEM), Sep. 2018, pp. $1458-1464$

[9] A. Carboni, K. El Shawarby, A. Di Gerlando, G. M. Foglia, R. Perini, and E. Ragaini, "Electric stress in power electronics applications," in 2019 XIII IEEE PES anchor conference in Europe (PowerTech), Milan, 2019. 